\title{
TiN-buffered substrates for photoelectrochemical measurements of oxynitride thin films
}

\author{
Markus Pichler ${ }^{\mathrm{a}}$, Daniele Pergolesi ${ }^{\mathrm{a}}$, Steve Landsmann ${ }^{\mathrm{b}}$, Vipin Chawla ${ }^{\mathrm{c}, \mathrm{d}}$, Johann \\ Michler $^{\mathrm{c}}$, Max Döbelie ${ }^{\mathrm{e}}$ Alexander Wokaun ${ }^{\mathrm{a}}$, Thomas Lipperta,f,* \\ ${ }^{a}$ Materials Group, General Energy Department, Paul Scherrer Institut, 5232 Villigen-PSI, \\ Switzerland \\ ${ }^{b}$ Empa, Swiss Federal Laboratories for Materials Science and Technology, Laboratory Materials for \\ Energy Conversion, Überlandstrasse 129, 8600 Dübendorf, Switzerland \\ ${ }^{c}$ Empa, Swiss Federal Laboratories for Materials Science and Technology, Laboratory for \\ Mechanics of Materials and Nanostructures, Feuerwerkerstrasse 39, 3602 Thun, Switzerland \\ ${ }^{d}$ Department of Physics, Maharishi Markandeshwar University, Mullana, 133207 Ambala, \\ Haryana, India \\ ${ }^{e}$ Ion Beam Physics, ETH Zurich, 8093 Zurich, Switzerland \\ ${ }^{f}$ Laboratory of Inorganic Chemistry, Department of Chemistry and Applied Biosciences, ETH \\ Zurich, 8093 Zurich, Switzerland
}

\section{Abstract}

Developing novel materials for the conversion of solar to chemical energy is becoming an increasingly important endeavour. Perovskite compounds based on bandgap tunable oxynitrides represent an exciting class of novel photoactive materials. To date, literature mostly focuses on the characterization of oxynitride powder samples which have undeniable technological interest but do not allow the investigation of fundamental properties such as the role of the crystalline quality and/or the surface crystallographic orientation toward photo-catalytic activity. The challenge of growing high quality oxynitride thin films arises from the availability of a suitable substrate, owing to strict material and processing requirements: effective lattice matching,

\footnotetext{
*Corresponding author

Email address: thomas.1ippert@psi.ch (Thomas Lippert)

$U R L:$ https://www.psi.ch/materials (Thomas Lippert)
} 
sufficiently high conductivities, stability under high temperatures and in strongly reducing environments. Here, we have established the foundations of a model system incorporating a TiN-buffer layer which enables fundamental investigations into crystallographic surface orientation and crystalline quality of the photocatalyst against photo(electro)chemical performance to be effectively performed. Furthermore, we find that TiN as current collector enables control over the nitrogen content of oxynitride thin films produced by a modified pulsed laser deposition method and allows the growth of highly ordered $\mathrm{LaTiO}_{3-\mathrm{x}} \mathrm{N}_{\mathrm{x}}$ thin films.

Keywords: Solar Water Splitting, Titanium Nitride, Oxynitride, Pulsed Laser Deposition, Photoelectrochemistry

2010 MSC: 00-01, 99-00 


\section{Introduction}

Due to the increasing demand of energy and the urgent need to reduce the amount of emitted greenhouse gases, intense research is carried out in the field of sustainable and renewable energy sources. Solar water splitting, also known as artificial pho-

5 tosynthesis, is among the most investigated routes for environmentally sustainable energy production. Artificial photosynthesis is based on the direct absorption of solar light by semiconductor materials with appropriate band gap matching the solar spectrum. The photo-generated electron-hole pairs drive (electro-)chemical reactions to split water into molecular oxygen and hydrogen. The latter can be used as energy carrier, for example in fuel cells.

Since the first report of $\mathrm{TiO}_{2}$ as photocatalyst by Fujishima and Honda in 1972[1], a great deal of research effort has been devoted to improving the performance as well as the discovery of new materials for photocatalytic water splitting. A plethora of oxides materials were found to be active for solar water splitting and were subject of further investigations, both experimentally [2, 3, 4, 5, 6] and theoretically [7, 8, 9, 10]. Also non-oxide compounds typically used for photovoltaic application were tested towards their activity for solar water splitting[11, 12, 13].

Oxides have typically a large band gap of more than $3 \mathrm{eV}$, therefore only absorbing UV radiation (about $5 \%$ of the solar spectrum)[14]. A greater portion of the solar spectrum can be captured by lowering the band gap, thus expanding the absorption window. Anion- and/or cation-doping is typically used to modify the electronic structure and as a consequence the optical properties of materials [15, 16, 17, 18, 19, 20]. For many oxides a reduced band gap can be achieved by substituting nitrogen into the oxygen site to form oxynitride materials $21,22,23,24,25,26$. The smaller band gap is the result of the hybridization of the $\mathrm{O} 2 \mathrm{p}$ and $\mathrm{N} 2 \mathrm{p}$ orbitals which is 
most likely to create additional available energy levels above the valence band of the original oxide, while leaving the conduction band unaffected. Density functional theory calculations for $\mathrm{BaTaO}_{2} \mathrm{~N}$ support this hypothesis[27].

However the size of the band gap of the photocatalyst is just one of the issues to be addressed to make solar water splitting efficient. Beside the chemical and structural stability in operating condition, the semiconductor used as photocatalyst should also offer high mobility of the photo-generated charge carriers to reduce charge recombination. Moreover, for the (electro-)chemical reaction to proceed, the band edges of the valence and conduction bands have to straddle both, the $\mathrm{O}_{2}$ and $\mathrm{H}_{2}$ evolution potentials. Fulfilling all these requirements remains a challenge.

Photocatalysts are usually characterized using powder samples [28, 29, 30, 20]. However, with powders it is difficult to probe specific material properties, such as the effect on the photocatalytic activity of the crystalline quality and/or surface crystallographic orientation. Moreover, in the specific case of oxynitride powders, the amount of incorporated nitrogen is usually set by the fabrication procedure which hampers the possibility of probing the effect of different nitrogen contents on the performance of the photocatalysts. Highly textured or epitaxial thin films can be used as model system to address these challenges.

Different experimental approaches are used to probe the photocatalytic activity of materials. Using powders one can measure directly the amount of gas produced as a consequence of specific photo-induced chemical reactions [24, 31]. Alternatively, the powders can be dispersed in aqueous solution with an organic dye that decomposes changing its absorbance as a result of the chemical reactions occurring at the photocatalyst under illumination [32, 33]. These techniques generally belong to the family of so-called photocatalytic characterizations. However, for the same illuminated area of the photocatalyst, the active surface area is orders of magnitude smaller 
using thin films compared to powders. As a consequence, the yield per unit area is much smaller for thin films than for powders hindering a direct photocatalytic characterization since the effect of the photo-induced reactions could be difficult to follow. Therefore, a photoelectrochemical (PEC) study is the only available technique to investigate thin film photocatalysts towards solar water splitting.

For PEC characterization the photocatalyst in contact with a current collector is immersed into an appropriate electrolyte solution and acts as the working electrode. A Pt wire is typically used as the counter electrode. In the typical 3-electrode configuration, the applied potentials between working and counter electrodes are referred to a reference electrode $(\mathrm{Ag} / \mathrm{AgCl}$ or hydrogen reference electrode). The photo-generated electrons and holes in the semiconducting photocatalyst reach the current collector and the interface with the electrolyte, respectively. The latter allow oxygen evolution at the photocatalyst surface producing positively charged ions that migrate into the electrolyte toward the working electrode. The electrons flow through an external circuit to the counter electrode where they enable hydrogen evolution. The measurement of the electronic photocurrent density (i.e. per unit of illuminated surface area) is a measure of the photocatalytic activity of the sample under investigation. Since relatively small current densities can be measured with high accuracy, this technique is very well suited for thin films studies if an appropriate conducting substrate or buffer layer for the growth of the photocatalyst is available.

In the literature only a few studies using thin films for PEC measurements have been reported, for example $\mathrm{Bi}_{2} \mathrm{FeCrO}_{6}$ to investigate the effect of the ferroelectric polarization on the photoelectrochemical performance [34] and $\mathrm{BiFeO}_{3}$ to test towards photoelectrochemical activity [35]. Concerning oxynitrides, thin films of these materials can be grown using different deposition methods, for example sputtering $\left(\mathrm{TiO}_{\mathrm{x}} \mathrm{N}_{\mathrm{y}}[15,36]\right.$ and $\left.\mathrm{LaTiO}_{3-\mathrm{x}} \mathrm{N}_{\mathrm{x}}[37]\right)$, pulsed laser deposition $\left(\mathrm{LaTiO}_{3-\mathrm{x}} \mathrm{N}_{\mathrm{x}}[38]\right.$, 
$\left.\mathrm{TiO}_{\mathrm{x}} \mathrm{N}_{\mathrm{y}}[17], \mathrm{BaTaO}_{2} \mathrm{~N}[39]\right)$ and chemical vapor deposition $\left(\mathrm{TiO}_{\mathrm{x}} \mathrm{N}_{\mathrm{y}}\right.$ [40, 41]). However, the reported studies of their photocatalytic activity are quite rare. PEC meaparent conducting glasses as substrates [36]. The influence of the crystalline quality on the photo-activity of $\mathrm{LaTiO}_{3-\mathrm{x}} \mathrm{N}_{\mathrm{x}}$ was studied using $\mathrm{Nb}$-doped $\mathrm{SrTiO}_{3}$ as a substrate and $\mathrm{IrO}_{2}$ as co-catalyst 37]. In both cases, reactive magnetron sputtering was applied to grow the oxynitride films.

For this work, a modified pulsed laser deposition method was selected to further investigate the effect of the nitrogen content and different surface orientations on the PEC performance. Based on our previous studies, highly ordered oxynitride thin films with different nitrogen content can be grown starting from a target of the original oxide, thus making this method potentially applicable to any oxynitride material circumventing the need of an oxynitride target whose preparation is often difficult [38, 42]. Additionally, this technique allows a systematic variation of the nitrogen content in the films, which was quantitatively investigated to minimize the presence of potential anionic vacancies.

However using this method, the strongly reducing environment and the high temperature needed for a sufficient incorporation of nitrogen into the oxynitride thin film [37, 38], preclude the use of several materials as current collector. This study aims to identify a stable and conducting material that allows the epitaxial growth of oxynitride photocatalyst thin films for PEC characterization.

As representative example of oxynitride materials for the photocatalyst, the orthorhombic perovskite $\mathrm{LaTiO}_{3-\mathrm{x}} \mathrm{N}_{\mathrm{x}}(0<x<1)$ is selected[43]. 


\section{Experimental section}

\subsection{Oxynitride thin film deposition}

$\mathrm{LaTiO}_{3-\mathrm{x}} \mathrm{N}_{\mathrm{x}}$ thin films have been grown on different substrates using a modified pulsed laser deposition (PLD) method called Pulsed Reactive Crossed-beam Laser

105

Ablation (PRCLA). During PRCLA suitable gas jets are injected into the vacuum chamber through a nozzle valve triggered by the laser pulses and crossing the ablation plume near the surface of the target. The specific features of this technique are described in details elsewhere [44], as well as a comparative study of the unique characteristics that distinguish PRCLA from conventional PLD[45]. Briefly, during PRCLA pulsed gas jets synchronized with the laser pulses are injected through a piezoelectric nozzle valve into the deposition chamber near the ablation spot at the target. Here the gas jets cross the ablation plume. By appropriately tuning the delay time (with respect to the laser pulse) and the opening time of the nozzle valve, it is possible to create a temporary strong pressure gradient along the direction of the expansion of the ablated material. The relatively high pressure at the very early stage of the propagation of the ablated species allows efficient physicochemical interaction with the gaseous environment. Moving away from the target toward the substrate the partial pressure rapidly decreases hindering further physicochemical evolution of the plasma species. Using an oxide target of $\mathrm{La}_{2} \mathrm{Ti}_{2} \mathrm{O}_{7}$ and $\mathrm{NH}_{3}$ for the pulsed gas jets, nitrogen can effectively be substituted into the oxygen sites allowing the growth of $\mathrm{LaTiO}_{3-\mathrm{x}} \mathrm{N}_{\mathrm{x}}$ thin films 38 .

For this work, a $\mathrm{La}_{2} \mathrm{Ti}_{2} \mathrm{O}_{7}$ target for laser ablation was prepared in our laboratory as a sintered ceramic rod. The vacuum chamber was evacuated to a base pressure of about $10^{-6}$ mbar. For $\mathrm{LaTiO}_{3-\mathrm{x}} \mathrm{N}_{\mathrm{x}}$ deposition a $\mathrm{N}_{2}$ background partial pressure of $8.0 \times 10^{-4}$ mbar was set and $\mathrm{NH}_{3}$ was used for the pulsed gas jets. A delay time 
of $30 \mu \mathrm{s}$ was set between the opening of the nozzle valve and the laser pulse, while the opening time of the nozzle valve was $400 \mu \mathrm{s}$ for each laser pulse. The laser (KrF excimer laser with $\lambda=248 \mathrm{~nm}$ and pulse width of $30 \mathrm{~ns}$ ) was operated at a repetition rate of $10 \mathrm{~Hz}$. In this condition, the average pressure in the vacuum chamber raises from the $\mathrm{N}_{2}$ background pressure up to about $1.8 \times 10^{-3}$ mbar. $\operatorname{LaTiO}_{3-\mathrm{x}} \mathrm{N}_{\mathrm{x}}$ thin films were fabricated using different laser fluences from $2 \mathrm{~J} \mathrm{~cm}^{-2}$ to $4 \mathrm{~J} \mathrm{~cm}^{-2}$ on a spot size of about $1 \mathrm{~mm}^{2}$ with a target-to-substrate distance of $50 \mathrm{~mm}$. Single crystal (100)-oriented $\mathrm{SrTiO}_{3}$ and $\mathrm{LaAlO}_{3}$, and (110) $\mathrm{YAlO}_{3}$ were used as substrates. The substrates were clamped on a Si plate with the same size, which was then heated resistively up to $870^{\circ} \mathrm{C}$. The temperature was measured with a pyrometer using 0.7 as the value of emissivity.

\subsection{The current collector}

For photoelectrochemical (PEC) characterization an electronic conducting substrate or buffer layer is required for the growth of $\mathrm{LaTiO}_{3-\mathrm{x}} \mathrm{N}_{\mathrm{x}}$. Beside the conducting substrate Nb-doped $\mathrm{SrTiO}_{3}$ used in [37] for the growth of oxynitride films by sputtering, the use of thin $\mathrm{SrRuO}_{3}$ and TiN buffer layers was investigated.

$\mathrm{SrRuO}_{3}$ thin films were grown by PLD on (100)-oriented $\mathrm{SrTiO}_{3}$. For the deposition, an $\mathrm{O}_{2}$ partial pressure of 0.1 mbar was set. A $\mathrm{SrRuO}_{3}$ disc target was ablated using the $\mathrm{KrF}$ excimer laser at a repetition rate of $4 \mathrm{~Hz}$ and a laser fluence of about $1.8 \mathrm{~J} \mathrm{~cm}^{-2}$. The target-to-substrate distance was $50 \mathrm{~mm}$ and the substrate was heated up to $800^{\circ} \mathrm{C}$.

TiN thin films were grown by DC reactive sputtering (Mantis Deposition Ltd, UK, Model: QPrep) of a Ti target in an $\mathrm{Ar} / \mathrm{N}_{2}$ gas mixture. (100)-oriented $\mathrm{MgO}$ substrates were used setting a temperature of $700^{\circ} \mathrm{C}$ of the heating stage. From a base pressure of $2.0 \times 10^{-7}$ mbar, gas flows of 35 and $15 \mathrm{sccm}$ of $\mathrm{Ar}$ and $\mathrm{N}_{2}$ respec- 
tively were let into the vacuum chamber reaching a pressure of $2.9 \times 10^{-3} \mathrm{mbar}$. The sputtering process was performed by applying an accelerating voltage of $446 \mathrm{~V}$ which set a current of about $300 \mathrm{~mA}$.

$\mathrm{LaTiO}_{3-\mathrm{x}} \mathrm{N}_{\mathrm{x}}$ thin films were then grown by PRCLA on the previously mentioned the photocurrent and a scan rate of $10 \mathrm{mV} \mathrm{s}^{-1}$ was used for linear scan voltammetry measurements. 


\subsection{Thin film characterizations}

175

The chemical composition of the thin films was determined by Rutherford Backscattering measurements using a $2 \mathrm{MeV}{ }^{4} \mathrm{He}$ beam and a silicon PIN diode detector under an angle of $168^{\circ}$ towards the incident beam. The collected data were analyzed using the RUMP program[47]. The nitrogen-to-oxygen ratio was determined by Heavy-Ion Elastic Recoil Detection Analysis (ERDA) using a $13 \mathrm{MeV}$ ${ }^{127}$ I beam and a combination of a time-of-flight spectrometer with a gas ionization detector [48]. In the composition depth profile a region between $20 \mathrm{~nm}$ and $100 \mathrm{~nm}$ was chosen for integration of the oxygen and nitrogen signal. The surface and the interface to the conducting substrate or buffer layer were carefully excluded. The crystalline properties were obtained using a Bruker-Siemens D500 X-Ray diffractometer. Transmittance data were collected with a Cary 500 Scan UV-Vis-NIR spectrophotometer in the wavelength range of $200 \mathrm{~nm}$ to $1000 \mathrm{~nm}(6.20 \mathrm{eV}$ to $1.24 \mathrm{eV})$. For the calibration of the deposition rates, the thicknesses of the samples were measured by surface profilometry (Dektak 8, Veeco Instruments). The surface morphology was investigated using a Zeiss Supra VP55 Scanning Electron Microscope with an in-lens detector. 


\section{Results and discussion}

\subsection{The current collector}

To collect the photo-generated charge carriers, the photocatalyst has to be in contact with an electronic conducting electrode. The material used for such a cur-

195 rent collector has to be chemically stable during the oxynitride film growth, i.e. at temperatures as high as about $800^{\circ} \mathrm{C}$ and in $\mathrm{NH}_{3}$ environment. To enable the investigation of the potential effects of the crystalline properties and surface orientation on the PEC performance, the conducting material has to be lattice matched for an epitaxial growth of the oxynitride thin film with good crystalline quality.

Two different experimental approaches can be followed, either the use of a conductive substrate or the use of a conducting thin film as seed layer for the growth of the oxynitride photocatalyst.

Among the suitable electronic conducting substrates, single crystal Nb-doped $\mathrm{SrTiO}_{3}(\mathrm{STO})$ show very good lattice matching to $\mathrm{LaTiO}_{3-\mathrm{x}} \mathrm{N}_{\mathrm{x}}$ (the STO lattice parameter is about $3.90 \AA$ (ICSD Coll.Code: 94573), while for $\mathrm{LaTiO}_{2} \mathrm{~N}$ the lattice constant is in the range of $3.95 \AA$ (ICSD Coll.Code: 168551), which can vary depending on the nitrogen content). These substrates were successfully used in Ref. [37] for the growth and characterization of epitaxial $\mathrm{LaTiO}_{3-\mathrm{x}} \mathrm{N}_{\mathrm{x}}$ films made by sputtering using a target of the cold pressed oxynitride powders. With our experimental conditions, it was not possible to obtain single phase $\mathrm{LaTiO}_{3-\mathrm{x}} \mathrm{N}_{\mathrm{x}}$ thin films on Nb-doped or un-doped STO substrates by PRCLA. The thin films grown on STO were (001) epitaxially oriented with the substrates, but the $\mathrm{LaTiO}_{3-\mathrm{x}} \mathrm{N}_{\mathrm{x}}$ diffraction reflexes appear as the result of the overlapping of multiple reflexes, as can be seen for example in the (400) diffraction reflex of $\mathrm{LaTiO}_{3-\mathrm{x}} \mathrm{N}_{\mathrm{x}}$ in Fig. 1. This may be explained due to the presence of multiple phases of $\mathrm{LaTiO}_{3-\mathrm{x}} \mathrm{N}_{\mathrm{x}}$ layers with different nitrogen content 
in the thin film. It has been shown that STO substrates can easily lose oxygen in reducing environment and exchange oxygen with the film growing on its surface [49]. The substrate itself can indeed provide a large part of the oxygen content of the growing film. When an oxynitride film grows on STO, this effect is particularly detrimental since oxygen atoms from the substrate can be incorporated into the film hindering the nitrogen substitution. In addition, the Nb-doped STO substrates showed strongly reduced conductivity after the oxynitride deposition, suggesting a decomposition of this material under the film growth conditions differently to what was observed for sputtering[37].

Concerning the use of conducting seed layers, for the conventional characterization of powder samples glass substrates coated with transparent conducting oxides are used to collect the photo-generated charge carriers. Typical coatings are made of aluminum-doped zinc oxide, fluorine-doped tin oxide or indium tin oxide. Polycrystalline TiON films were grown by sputtering at relatively low temperature $\left(>250{ }^{\circ} \mathrm{C}\right)$ on these kind of conducting glasses[36]. These commercially available substrates are amorphous, thus only polycrystalline films can be obtained. Moreover, at the high temperatures required for the growth of oxynitride films with high crystalline quality $\left(>700^{\circ} \mathrm{C}\right)$, transparent conducting oxides undergo a thermal decomposition and their conductivity significantly decreases[50]. This makes these materials unsuitable for our purpose even if coatings with high crystalline quality were used.

Thin films of non-transparent electronic conductive oxides could also be used as current collectors. Unfortunately, most of the electronic conducting oxides show suitable electronic conductivity only at relatively high temperatures. But the main problem here is that we could not find any electronic conducting oxide able to provide the required physicochemical stability during the oxynitride growth condition using PRCLA of the native oxide target, i.e. a strongly reducing environment at high 
temperatures. Literature reports that $\mathrm{SrRuO}_{3}$-buffered substrates were used for the growth of $\mathrm{BaTaO}_{3-\mathrm{x}} \mathrm{N}_{\mathrm{x}}[39]$. This was achieved by conventional PLD using the oxynitride material as the target and an $\mathrm{O}_{2} / \mathrm{N}_{2}$ gas mixture, i.e. in oxygen-containing environment. For this work we also have attempted the use of $\mathrm{SrRuO}_{3}$. Thin films of this material were epitaxially grown on STO substrates at $>800{ }^{\circ} \mathrm{C}$ in an oxygen partial pressure of 0.1 mbar. After XRD analysis, the as-grown films were heated up in vacuum at a temperature of about $600^{\circ} \mathrm{C}$. Such a thermal treatment in vacuum was enough to decompose the $\mathrm{SrRuO}_{3}$ film, as revealed by XRD analysis, confirming the poor stability of this material in low oxygen-containing environment at high temperature, as already reported in the literature [51, 52]. In the specific case of $\mathrm{LaTiO}_{3-\mathrm{x}} \mathrm{N}_{\mathrm{x}}$, a sufficient nitogen substitution by PRCLA using the native oxide target requires a gaseous environment with very low oxygen partial pressure (using $\mathrm{N}_{2}$ and $\mathrm{NH}_{3}$ ). This precludes the use of $\mathrm{SrRuO}_{3}$ as seed layer.

255 Other possible alternative current collectors are thin films of noble metals, such as $\mathrm{Pt}$ or $\mathrm{Au}$, which also have the advantage of a cubic crystalline structure with lattice parameter similar to that of $\mathrm{LaTiO}_{3-\mathrm{x}} \mathrm{N}_{\mathrm{x}}$ (3.92 $\AA$ for Pt (ICSD Coll.Code: 180880), $4.08 \AA$ for Au (ICSD Coll.Code: 52700), and about $3.95 \AA$ for $\mathrm{LaTiO}_{2} \mathrm{~N}$ (ICSD Coll.Code: 168551), as an example). However, at the high temperatures required for the oxynitride growth, noble metals show very poor adhesion on the polished surface of single crystal substrates [53] and typically dewet the substrate surface. The use of additional adhesion layers (typically Ti or Cr are used) would be necessary. Beside the additional complication of the growth of a further layer, the typical metals used to aids $\mathrm{Pt}$ or $\mathrm{Au}$ adhesion show different crystalline structures compare to $\mathrm{Pt}$ and $\mathrm{Au}$, thus precluding the growth of current collectors with high crystalline quality which is a fundamental prerequisite for the growth of high quality epitaxial oxynitride films. 
In a different approach, the group of metal nitrides comprises electronically conducting compounds that are known to be chemically very stable over a wide range on $\mathrm{MgO}$ substrates via an almost continuous line of interfacial misfit dislocations capable of adjusting a lattice mismatch as large as $29 \%$. 


\subsection{The growth of the oxynitride photocatalyst on the selected current collector}

295 $\operatorname{sis}(\mathrm{ERDA})$.

Fig. 2c shows an RBS spectrum of a $\mathrm{LaTiO}_{3-\mathrm{x}} \mathrm{N}_{\mathrm{x}}$ film. At high energies, the first peak corresponds to La, the heavy element, present in the oxynitride film. At intermediate energies, signals from Ti were detected and since Ti is in both layers, a 

derstand up to which extent the nitrogen content could be tuned in our films. Previous studies on $\mathrm{LaTiO}_{3-\mathrm{x}} \mathrm{N}_{\mathrm{x}}$ film growth by PRCLA showed that high laser fluence and high deposition temperatures are required to achieve high nitrogen substitution[38]. For this work we systematically investigated the effect of the laser fluence and sub- 
strate material on the $N / O$ ratio as determined by ERDA for several $\mathrm{LaTiO}_{3-\mathrm{x}} \mathrm{N}_{\mathrm{x}}$ films. For this investigation the laser fluence was varied from $2 \mathrm{~J} \mathrm{~cm}^{-2}$ to $4 \mathrm{~J} \mathrm{~cm}^{-2}$ and (100)-oriented $\mathrm{SrTiO}_{3}(\mathrm{STO})$ and $\mathrm{LaAlO}_{3}(\mathrm{LAO}),(110)$-oriented $\mathrm{YAlO}_{3}(\mathrm{YAO})$, as well as (100)-oriented TiN-coated $\mathrm{MgO}$ substrates were used. Following this approach, the typical composition of the oxynitride thin films determined by RBS and ERDA can be written as $\mathrm{La}_{1+\mathrm{y}} \mathrm{Ti}_{1-\mathrm{y}} \mathrm{O}_{3-\mathrm{x}} \mathrm{N}_{\mathrm{z}}$ where $0<y<0.03$ and $0.5<x<0.85$, depending on deposition parameters. Further, the sum of the oxygen and nitrogen content is close to $3(x \approx z)$, thus forming the $\mathrm{LaTiO}_{3-\mathrm{x}} \mathrm{N}_{\mathrm{x}}$ perovskite with a very small amount of anionic vacancies.

In particular, Fig. 3 shows the measured $N / O$ ratio as a function of laser fluence for the different substrates. As a first observation, it appears clearly that a TiN-buffered substrate allows a more reliable control of the nitrogen content by only changing the laser fluence. The points corresponding to TiN-buffered $\mathrm{MgO}$ show a linear increase of the $N / O$ ratio with increasing fluence (from $2.5 \mathrm{~J} \mathrm{~cm}^{-2}$ to $4.1 \mathrm{~J} \mathrm{~cm}^{-2}$ ). The points corresponding to the oxide substrates are more scattered. As mentioned previously, STO substrates do not preserve their oxygen content at high temperatures and in reducing environment. Oxygen atoms from the substrate can even migrate into the growing film hindering the nitrogen substitution. In fact, a clear dependence of the $N / O$ ratio on the laser fluence using STO substrates is not visible, neither at low nor at high fluence. Using the other oxide substrates, YAO and LAO, we observed a trend similar to that observed for TiN-buffered $\mathrm{MgO}$ at lower fluences $\left(2.0 \mathrm{~J} \mathrm{~cm}^{-2}\right.$ to $\left.3.0 \mathrm{~J} \mathrm{~cm}^{-2}\right)$, but with increasing fluence the values of $N / O$ ratio measured for the $\operatorname{LaTiO}_{3-\mathrm{x}} \mathrm{N}_{\mathrm{x}}$ films are much more scattered. More importantly, the maximum achievable nitrogen content is lower when the oxynitride films grow directly on the oxide surfaces than on the TiN-coated substrates. The effect of oxygen diffusion into the film as described 
before for STO, is also known for LAO substrates [49. This suggests that with YAO substrates such a mechanism may also apply, but it is less significant when using YAO or LAO instead of STO. However, the possible presence of a gradient in the nitrogen content of the film along the growth direction should be taken into account. Fig. 3 shows that to achieve a better control of the nitrogen content and a larger amount of nitrogen substitution in the oxynitride film a nitride-coated substrate like TiN-buffered $\mathrm{MgO}$ is preferable and underlines the advantage of the sample design proposed here for the PEC characterization of oxynitride photocatalysts.

Fig. 4 shows the effect of different nitrogen contents on the optical properties of the $\mathrm{LaTiO}_{3-\mathrm{x}} \mathrm{N}_{\mathrm{x}}$ films. For this measurement double-side polished LAO substrates were used to acquire UV-Vis spectra in transmission mode. The transmittance is plotted as a function of the incident wavelength showing a shift of the band gap towards longer wavelengths with increasing nitrogen content, as expected. Out of the UV-Vis spectra a band gap of about $2.35 \mathrm{eV}$ to $2.85 \mathrm{eV}$ can be estimated for the oxynitride thin films 38. For comparison, the UV-Vis spectrum of a thin film of $\mathrm{La}_{2} \mathrm{Ti}_{2} \mathrm{O}_{7}$ grown on LAO by conventional PLD is also shown.

\subsection{Tuning the crystallographic properties}

Concerning the crystalline quality and crystallographic orientation of the oxynitride films grown on (100)-oriented TiN-buffered $\mathrm{MgO}$ we observed a remarkable effect of the laser fluence. The polycrystalline film discussed in Fig. 2 were grown using a laser fluence of about $3.7 \mathrm{~J} \mathrm{~cm}^{-2}$. By keeping all other deposition parameters constant but lowering the fluence to $3.0 \mathrm{~J} \mathrm{~cm}^{-2}$, the $\mathrm{LaTiO}_{3-\mathrm{x}} \mathrm{N}_{\mathrm{x}}$ thin films grew epitaxially oriented with the substrate, as shown in Fig. 5. In this case, it comes at the cost of a lower nitrogen content. As shown in Fig. 3, for a laser fluence of about

$3.0 \mathrm{~J} \mathrm{~cm}^{-2}$ the $N / O$ ratio is about 0.30 , while 0.36 was the value measured for a laser 
fluence of about $3.7 \mathrm{~J} \mathrm{~cm}^{-2}$, though in the second case only polycrystalline samples could be grown.

This experimental observation could be explained in analogy with the equivalent effect often encountered during conventional PLD; by increasing the laser fluence both the kinetic energy of the ablated species and the deposition rate increase. Above a certain threshold this may hinder the ordered growth of the film. Literature on PRCLA is scarce and we cannot refer to any specific examples to support this speculation but it is likely to assume the same mechanism to apply also for PRCLA.

While higher laser fluence favors the growth of polycrystalline thin films with higher nitrogen content, reduced laser fluence leads to an epitaxial film growth with lower nitrogen content. A similar effect was observed for $\mathrm{LaTiO}_{3-\mathrm{x}} \mathrm{N}_{\mathrm{x}}$ films grown by reactive radio-frequency sputtering [37]. The $\mathrm{N} / \mathrm{O}$ ratio was estimated by coupled energy-dispersive spectroscopy. Films with higher nitrogen content were polycrystalline while those with lower nitrogen content were epitaxially grown.

A comparative investigation of the effect of the surface orientation and crystalline quality on PEC performance should be performed using films with similar nitrogen content. This could be achieved by keeping the laser fluence within a range that promotes the epitaxial highly ordered growth on TiN-buffered MgO but changing the substrate material and/or deposition parameters, such as substrate temperature, in order to grow TiN layers with different orientations or as polycrystalline seed layers for the growth of the oxynitride films. The oxynitride films will thus show different crystallographic and morphological properties but their nitrogen content will be comparable with that of the highly ordered epitaxial film. 


\subsection{Proof of concept of the model system using photoelectrochemical characterization}

As a preliminary test experiment to validate our sample design, the polycrystalline $\mathrm{La}_{1.01} \mathrm{Ti}_{0.99} \mathrm{O}_{2.2} \mathrm{~N}_{0.8}$ sample grown on TiN-buffered $\mathrm{MgO}$ substrate was characterized in a PEC setup. The photocurrent generated under the effect of Xenon lamp front-illumination was measured while sweeping the applied voltage from $0.2 \mathrm{~V}$ to $1.4 \mathrm{~V}$ versus the reversible hydrogen electrode (RHE). A rotating shutter was used to chop the light allowing the simultaneous measurement of the so-called dark current (no illumination of the sample). ERDA analysis showed that a small but measurable amount of oxygen is present in the TiN current collector (N/O ratio $>6.5$ ). In order to exclude any contribution to the photocurrent from the buried TiN layer, a PEC measurement was performed using a bare TiN-buffered MgO sample. As expected no photoresponse was detected.

For an unoptimized system the result of our preliminary PEC measurements for $\mathrm{La}_{1.01} \mathrm{Ti}_{0.99} \mathrm{O}_{2.2} \mathrm{~N}_{0.8}$ is promising, as per the linear voltammetry measurements shown in Fig. 6. We note that the measured photocurrents are lower than what has been reported for powder based oxynitrides, this is to be expected due to the difference in surface-to-volume ratio (or the extent of the electrochemically active area). Furthermore, the specific morphology of this sample characterized by extended grain boundary regions where untimely recombination of the photo-generated charge carriers is strongly favored likely represents a possible loss channel. We anticipate that by employing sacrificial reagents acting as hole scavengers or co-catalysts instead of just the semiconductor in direct contact with the electrolyte, will improve the performance 37]. Finally, the very broad absorption edge of this sample as shown in Fig. 4 may negatively influence the values of the photocurrent. 


\section{Conclusion}

In summary we report a new experimental approach which, starting from the ship between crystallographic properties and electrochemical response is expected to aid the development and design of better performing materials for solar-driven water splitting. 


\section{Acknowledgment}

We would like to thank the Paul Scherrer Institut and the Swiss National Science Foundation (IZERZ0_142176) for financial supports. 


\section{References}

[1] A. FUJISHIMA, K. HONDA, Electrochemical Photolysis of Water at a Semiconductor Electrode, Nature 238 (5358) (1972) 37-38. doi:10.1038/238037a0.

[2] A. Kudo, Y. Miseki, Heterogeneous photocatalyst materials for water splitting., Chemical Society reviews 38 (1) (2009) 253-78. doi:10.1039/b800489g.

[3] K. Maeda, Photocatalytic water splitting using semiconductor particles: History and recent developments, Journal of Photochemistry and Photobiology C: Photochemistry Reviews 12 (4) (2011) 237-268. doi:10.1016/j.jphotochemrev. 2011.07 .001 .

[4] X. Liu, F. Wang, Q. Wang, Nanostructure-based WO3 photoanodes for photoelectrochemical water splitting., Physical chemistry chemical physics : PCCP 14 (22) (2012) 7894-911. doi:10.1039/c2cp40976c.

[5] K. Sivula, F. Le Formal, M. Grätzel, Solar water splitting: progress using hematite $(\alpha-\mathrm{Fe}(2) \mathrm{O}(3))$ photoelectrodes., ChemSusChem 4 (4) (2011) 432 49. doi:10.1002/cssc.201000416.

[6] S. Cho, J.-W. Jang, K.-H. Lee, J. S. Lee, Research Update: Strategies for efficient photoelectrochemical water splitting using metal oxide photoanodes, APL Materials 2 (1) (2014) 010703. doi:10.1063/1.4861798.

[7] I. E. Castelli, T. Olsen, S. Datta, D. D. Landis, S. r. Dahl, K. S. Thygesen, K. W. Jacobsen, Computational screening of perovskite metal oxides for optimal solar light capture, Energy \& Environmental Science 5 (2) (2012) 5814. doi: 10.1039/c1ee02717d. 
[8] I. E. Castelli, D. D. Landis, K. S. Thygesen, S. r. Dahl, I. Chorkendorff, T. F. Jaramillo, K. W. Jacobsen, New cubic perovskites for one- and two-photon water splitting using the computational materials repository, Energy \& Environmental Science 5 (10) (2012) 9034. doi:10.1039/c2ee22341d.

[9] W. Li, E. Ionescu, R. Riedel, A. Gurlo, Can we predict the formability of perovskite oxynitrides from tolerance and octahedral factors?, Journal of Materials Chemistry A 1 (39) (2013) 12239. doi:10.1039/c3ta10216e.

[10] A. K. Singh, K. Mathew, H. L. Zhuang, R. G. Hennig, Computational Screening of 2D Materials for Photocatalysis, The Journal of Physical Chemistry Letters 6 (6) (2015) 1087-1098. doi:10.1021/jz502646d.

[11] D. Yokoyama, T. Minegishi, K. Jimbo, T. Hisatomi, G. Ma, M. Katayama, J. Kubota, H. Katagiri, K. Domen, H 2 Evolution from Water on Modified Cu 2 ZnSnS 4 Photoelectrode under Solar Light, Applied Physics Express 3 (10) (2010) 101202. doi:10.1143/APEX .3.101202.

[12] L. Rovelli, S. D. Tilley, K. Sivula, Optimization and stabilization of electrodeposited $\mathrm{Cu} 2 \mathrm{ZnSnS4}$ photocathodes for solar water reduction., ACS applied materials \& interfaces 5 (16) (2013) 8018-24. doi:10.1021/am402096r.

[13] W. Septina, S. Ikeda, T. Harada, M. Higashi, R. Abe, M. Matsumura, Photosplitting of Water from Wide-Gap $\mathrm{Cu}(\mathrm{In}, \mathrm{Ga}) \mathrm{S} 2$ Thin Films Modified with a CdS Layer and Pt Nanoparticles for a High-Onset-Potential Photocathode, The Journal of Physical Chemistry C 119 (16) (2015) 8576-8583. doi: 10.1021/acs.jpcc.5b02068. 
[14] D. Scaife, Oxide semiconductors in photoelectrochemical conversion of solar energy, Solar Energy 25 (1) (1980) 41-54. doi:10.1016/0038-092X (80)90405-3.

[15] H. Fakhouri, J. Pulpytel, W. Smith, A. Zolfaghari, H. R. Mortaheb, F. Meshkini,

[19] N. Serpone, Is the band gap of pristine $\mathrm{TiO}(2)$ narrowed by anion- and cationdoping of titanium dioxide in second-generation photocatalysts?, The journal of physical chemistry. B 110 (48) (2006) 24287-93. doi:10.1021/jp065659r.

[20] M. Higashi, Y. Yamanaka, O. Tomita, R. Abe, Fabrication of cation-doped 

$\mathrm{BaTaO} 2 \mathrm{~N}$ photoanodes for efficient photoelectrochemical water splitting under visible light irradiation, APL Materials 3 (10) (2015) 104418. doi:10.1063/1. 4931487.

[21] T. Takata, C. Pan, K. Domen, Recent progress in oxynitride photocatalysts for visible-light-driven water splitting, Science and Technology of Advanced Materials 16 (3) (2015) 033506. doi:10.1088/1468-6996/16/3/033506.

[22] K. Maeda, K. Domen, Oxynitride materials for solar water splitting, MRS Bulletin 36 (01) (2011) 25-31. doi:10.1557/mrs.2010.4.

[23] K. Maeda, K. Domen, Solid Solution of GaN and ZnO as a Stable Photocatalyst for Overall Water Splitting under Visible Light, Chemistry of Materials 22 (3) (2010) 612-623. doi:10.1021/cm901917a.

[24] A. Kasahara, K. Nukumizu, T. Takata, J. N. Kondo, M. Hara, H. Kobayashi, K. Domen, LaTiO $2 \mathrm{~N}$ as a Visible-Light (600 nm)-Driven Photocatalyst (2), The Journal of Physical Chemistry B 107 (3) (2003) 791-797. doi:10.1021/ jp026767q.

[25] K. Maeda, D. Lu, K. Domen, Direct water splitting into hydrogen and oxygen under visible light by using modified $\mathrm{TaON}$ photocatalysts with $\mathrm{d}(0)$ electronic configuration., Chemistry (Weinheim an der Bergstrasse, Germany) 19 (16) (2013) 4986-91. doi:10.1002/chem.201300158.

[26] S. G. Ebbinghaus, H.-P. Abicht, R. Dronskowski, T. Müller, A. Reller, A. Weidenkaff, Perovskite-related oxynitrides Recent developments in synthesis, characterisation and investigations of physical properties, Progress in Solid State 
Chemistry 37 (2) (2009) 173-205. doi:10.1016/j.progsolidstchem.2009. 11.003 .

[27] K. Maeda, K. Domen, New Non-Oxide Photocatalysts Designed for Overall Water Splitting under Visible Light, Journal of Physical Chemistry C 111 (22) (2007) 7851-7861. doi:10.1021/jp070911w.

[28] R. B. Singh, H. Matsuzaki, Y. Suzuki, K. Seki, T. Minegishi, T. Hisatomi, K. Domen, A. Furube, Trapped state sensitive kinetics in LaTiO2N solid photocatalyst with and without cocatalyst loading., Journal of the American Chemical Society 136 (49) (2014) 17324-31. doi:10.1021/ja5102823.

[29] S. Landsmann, A. E. Maegli, M. Trottmann, C. Battaglia, A. Weidenkaff, S. Pokrant, Design Guidelines for High-Performance Particle-Based Photoanodes for Water Splitting: Lanthanum Titanium Oxynitride as a Model., ChemSusChemdoi:10.1002/cssc.201500830.

[30] N. Nishimura, B. Raphael, K. Maeda, L. Le Gendre, R. Abe, J. Kubota, K. Domen, Effect of TiCl4 treatment on the photoelectrochemical properties of LaTiO2N electrodes for water splitting under visible light, Thin Solid Films 518 (20) (2010) 5855-5859. doi:10.1016/j.tsf.2010.05.094.

[31] D. YAMASITA, Recent progress of visible-light-driven heterogeneous photocatalysts for overall water splitting, Solid State Ionics 172 (1-4) (2004) 591-595. doi:10.1016/j.ssi.2004.04.033.

[32] I. K. Konstantinou, T. A. Albanis, TiO2-assisted photocatalytic degradation of azo dyes in aqueous solution: kinetic and mechanistic investigations, Applied 
[33] B. Barrocas, O. Monteiro, M. M. Jorge, S. Sério, Photocatalytic activity and reusability study of nanocrystalline $\mathrm{TiO} 2$ films prepared by sputtering technique, [

[38] I. Marozau, A. Shkabko, M. Döbeli, T. Lippert, M. Mallepell, C. Schneider, 
A. Weidenkaff, A. Wokaun, Pulsed laser deposition and characterisation of perovskite-type LaTiO3xNx thin films, Acta Materialia 59 (19) (2011) 71457154. doi:10.1016/j.actamat.2011.08.019.

[39] Y.-I. Kim, W. Si, P. M. Woodward, E. Sutter, S. Park, T. Vogt, Epitaxial ThinFilm Deposition and Dielectric Properties of the Perovskite Oxynitride BaTaO 2 N, Chemistry of Materials 19 (3) (2007) 618-623. doi:10.1021/cm062480k.

[40] F. Fabreguette, L. Imhoff, J. Guillot, B. Domenichini, M. Marco de Lucas, P. Sibillot, S. Bourgeois, M. Sacilotti, Temperature and substrate influence on the structure of TiNxOy thin films grown by low pressure metal organic chemical vapour deposition, Surface and Coatings Technology 125 (1-3) (2000) 396-399. doi:10.1016/S0257-8972(99)00588-5.

[41] G. Hyett, M. A. Green, I. P. Parkin, The Use of Combinatorial Chemical Vapor Deposition in the Synthesis of Ti $3-\delta \mathrm{O} 4 \mathrm{~N}$ with $0.06 ; \delta ; 0.25$ : A Titanium Oxynitride Phase Isostructural to Anosovite, Journal of the American Chemical Society 129 (50) (2007) 15541-15548. doi:10.1021/ja073355s.

[42] I. Marozau, A. Shkabko, G. Dinescu, M. Döbeli, T. Lippert, D. Logvinovich, M. Mallepell, C. Schneider, A. Weidenkaff, A. Wokaun, Pulsed laser deposition and characterization of nitrogen-substituted SrTiO3 thin films, Applied Surface Science 255 (10) (2009) 5252-5255. doi:10.1016/j.apsusc.2008.07.159.

[43] M. Yashima, M. Saito, H. Nakano, T. Takata, K. Ogisu, K. Domen, Imma perovskite-type oxynitride LaTiO2N: structure and electron density., Chemical communications (Cambridge, England) 46 (26) (2010) 4704-6. doi:10.1039/ c0cc00573h. 
]

625

[44] P. Willmott, Pulsed reactive crossed-beam laser ablation, Applied Physics A: Materials Science \& Processing 69 (7) (1999) S437-S440. doi:10.1007/ s003399900354.

[45] J. Chen, D. Stender, M. Pichler, M. Döbeli, D. Pergolesi, C. W. Schneider, A. Wokaun, T. Lippert, Tracing the plasma interactions for pulsed reactive crossed-beam laser ablation, Journal of Applied Physics 118 (16) (2015) 165306. doi:10.1063/1.4934553.

[46] T. Lopes, L. Andrade, H. A. Ribeiro, A. Mendes, Characterization of photoelectrochemical cells for water splitting by electrochemical impedance spectroscopy, International Journal of Hydrogen Energy 35 (20) (2010) 11601-11608. doi:10.1016/j.ijhydene.2010.04.001.

[47] L. R. Doolittle, A semiautomatic algorithm for rutherford backscattering analysis, Nuclear Instruments and Methods in Physics Research Section B: Beam Interactions with Materials and Atoms 15 (1-6) (1986) 227-231. doi:10.1016/ 0168-583X (86) 90291-0.

[48] C. Kottler, M. Döbeli, F. Glaus, M. Suter, A spectrometer for low-energy heavy ion ERDA, Nuclear instruments \&amp; methods in physics research. Section B, Beam interactions with materials and atoms 248 (1) (2006) 155-162. doi: $10.1016 / j . n i m b .2006 .02 .013$.

[49] C. W. Schneider, M. Esposito, I. Marozau, K. Conder, M. Doebeli, Y. Hu, M. Mallepell, A. Wokaun, T. Lippert, The origin of oxygen in oxide thin films: Role of the substrate, Applied Physics Letters 97 (19) (2010) 192107. doi: $10.1063 / 1.3515849$. 
[50] R. van de Krol, M. Grätzel, Photoelectrochemical Hydrogen Production, Springer Science+Business Media, LLC, 233 Spring Street, New York, NY 10013, USA, 2012. doi:10.1007/978-1-4614-1380-6.

[51] H. N. Lee, H. M. Christen, M. F. Chisholm, C. M. Rouleau, D. H. Lowndes, Thermal stability of epitaxial $\mathrm{SrRuO}[\mathrm{sub} 3]$ films as a function of oxygen pressure, Applied Physics Letters 84 (20) (2004) 4107. doi:10.1063/1.1753650.

[52] J. Shin, S. Kalinin, H. Lee, H. Christen, R. Moore, E. Plummer, A. Baddorf, Surface stability of epitaxial SrRuO3 films, Surface Science 581 (2-3) (2005) 118-132. doi:10.1016/j.susc.2005.02.038.

[53] K. Mougin, Z. Zheng, N. Piazzon, E. Gnecco, H. Haidara, Thermal stability and reconstruction of nanoparticulate Au film on model molecular surfaces., Journal of colloid and interface science 333 (2) (2009) 719-24. doi:10.1016/j.jcis. 2009.01 .044$.

[54] D. Pergolesi, E. Fabbri, S. N. Cook, V. Roddatis, E. Traversa, J. A. Kilner, Tensile lattice distortion does not affect oxygen transport in yttria-stabilized 口 zirconia-CeO2 heterointerfaces., ACS nano 6 (12) (2012) 10524-34. doi:10. $1021 / \mathrm{nn} 302812 \mathrm{~m}$.

[55] S. Sanna, V. Esposito, D. Pergolesi, A. Orsini, A. Tebano, S. Licoccia, G. Balestrino, E. Traversa, Fabrication and Electrochemical Properties of Epitaxial Samarium-Doped Ceria Films on SrTiO 3 -Buffered MgO Substrates, 口 Advanced Functional Materials 19 (11) (2009) 1713-1719. doi:10.1002/adfm. 200801768 .

[56] A. Tiwari, J. Narayan, C. Jin, A. Kvit, Growth of epitaxial NdNiO[sub 3] and 
integration with $\mathrm{Si}(100)$, Applied Physics Letters 80 (8) (2002) 1337. doi: 10.1063/1.1451984.

[57] K. Wong, Y. Leung, Heteroepitaxial growth of La0.7Ca0.3MnO3/SrTiO3/TiN/Si by pulsed laser deposition, Thin Solid Films 354 (1-2) (1999) 55-58. doi:10.1016/S0040-6090(99) 00529-5.

[58] W. Wu, K. Wong, C. Choy, Epitaxial growth of SrTiO3 films with different orientations on TiN buffered $\mathrm{Si}(001)$ by pulsed laser deposition, Thin Solid Films 360 (1-2) (2000) 103-106. doi:10.1016/S0040-6090(99)01091-3.

[59] R. Pérez Casero, R. Gómez San Román, J. Perrière, A. Laurent, W. Seiler, 670 P. Gergaud, D. Keller, Epitaxial growth of $\mathrm{CeO} 2$ on $\mathrm{MgO}$ by pulsed laser 【 deposition, Applied Surface Science 109-110 (1997) 341-344. doi:10.1016/ S0169-4332(96) 00671-X.

[60] L. Chen, C. L. Chen, X. Chen, W. Donner, S. W. Liu, Y. Lin, D. X. Huang, A. J. Jacobson, Electrical properties of a highly oriented, textured thin film of the ionic conductor Gd:CeO[sub 2 $\delta]$ on (001) MgO, Applied Physics Letters 83 (23) (2003) 4737. doi:10.1063/1.1629378.

[61] R. Swanepoel, Determination of the thickness and optical constants of amorphous silicon, Journal of Physics E: Scientific Instruments 16 (12) (1983) 12141222. doi:10.1088/0022-3735/16/12/023. 


\section{List of Figures}

1 XRD patterns of two representative $\mathrm{LaTiO}_{3-\mathrm{x}} \mathrm{N}_{\mathrm{x}}$ samples grown on $\mathrm{SrTiO}_{3}$ substrates by PRCLA with different laser fluences. . . . . . . . . . . . . . . . . . . . . . . . . . . . . . 34

2 a) XRD pattern of a polycrystalline $\mathrm{La}_{1.01} \mathrm{Ti}_{0.99} \mathrm{O}_{2.2} \mathrm{~N}_{0.8}$ thin film deposited on TiN-buffered $\mathrm{MgO}$ substrate using PRCLA with a laser fluence of $3.7 \mathrm{~J} \mathrm{~cm}^{-2}$. The peak marked with an $\mathrm{X}$ is the $\mathrm{K}_{\beta}$ diffraction reflex from the $\mathrm{MgO}$ substrate. b) Cross section micrograph (fractured surface). c) The corresponding RBS spectrum (black points) analyzed with the RUMP program

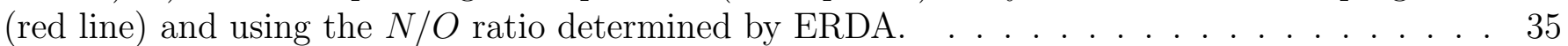

$3 \quad \mathrm{~N} / \mathrm{O}$ ratio of $\mathrm{LaTiO}_{3-\mathrm{x}} \mathrm{N}_{\mathrm{x}}$ thin films grown on different substrates as a function of laser fluence. Used substrates are $\mathrm{SrTiO}_{3}$ (blue 4), $\mathrm{YAlO}_{3}$ (black $\boldsymbol{\nabla}$ ), $\mathrm{LaAlO}_{3}$ (light green $\boldsymbol{\Delta}$ ), TiN-coated

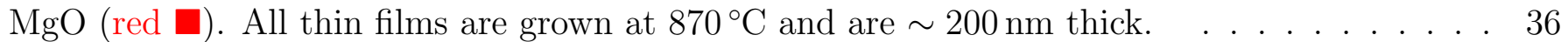

690

-

4 UV-Vis spectra of three $\mathrm{LaTiO}_{3-\mathrm{x}} \mathrm{N}_{\mathrm{x}}$ samples grown on $\mathrm{LaAlO}_{3}$ substrates with different nitrogen content showing the effect on the optical properties of nitrogen substitution into the native oxide. All samples are about $200 \mathrm{~nm}$ thick and therefore interference fringes are observed during the UV-Vis measurement[61]. For comparison, the transmittance spectrum of the $\mathrm{La}_{2} \mathrm{Ti}_{2} \mathrm{O}_{7}$ thin film of the same thickness is also shown. . . . . . . . . . . . . . . . . . . . . . . . . . 37

$5 \quad$ XRD pattern of the epitaxial (001)-oriented $\mathrm{La}_{1.04} \mathrm{Ti}_{0.96} \mathrm{O}_{2.26} \mathrm{~N}_{0.67}$ thin film grown by PRCLA with a laser fluence of $3.0 \mathrm{~J} \mathrm{~cm}^{-2}$. The peak marked with an $\mathrm{X}$ is the $\mathrm{K}_{\beta}$ diffraction reflex from the MgO substrate. $\ldots \ldots \ldots \ldots \ldots \ldots \ldots$

$6 \quad$ PEC measurement of the polycrystalline $\mathrm{La}_{1.01} \mathrm{Ti}_{0.99} \mathrm{O}_{2.2} \mathrm{~N}_{0.8}$ sample prepared on a TiN-buffered $\mathrm{MgO}$ substrate. The measurement was performed under chopped illumination $\left(100 \mathrm{~mW} \mathrm{~cm}^{-2}\right.$ with UV cutoff filter at $\lambda=420 \mathrm{~nm}$ ) to simultaneously measure the dark current and the photocurrent. The cell was filled with sodium borate electrolyte buffered at $\mathrm{pH}$ of 9 and a linear scan voltammetry was performed at a scan rate of $10 \mathrm{mV} \mathrm{s}^{-1}$. . . . . . . . . . . . . 39 
Figures

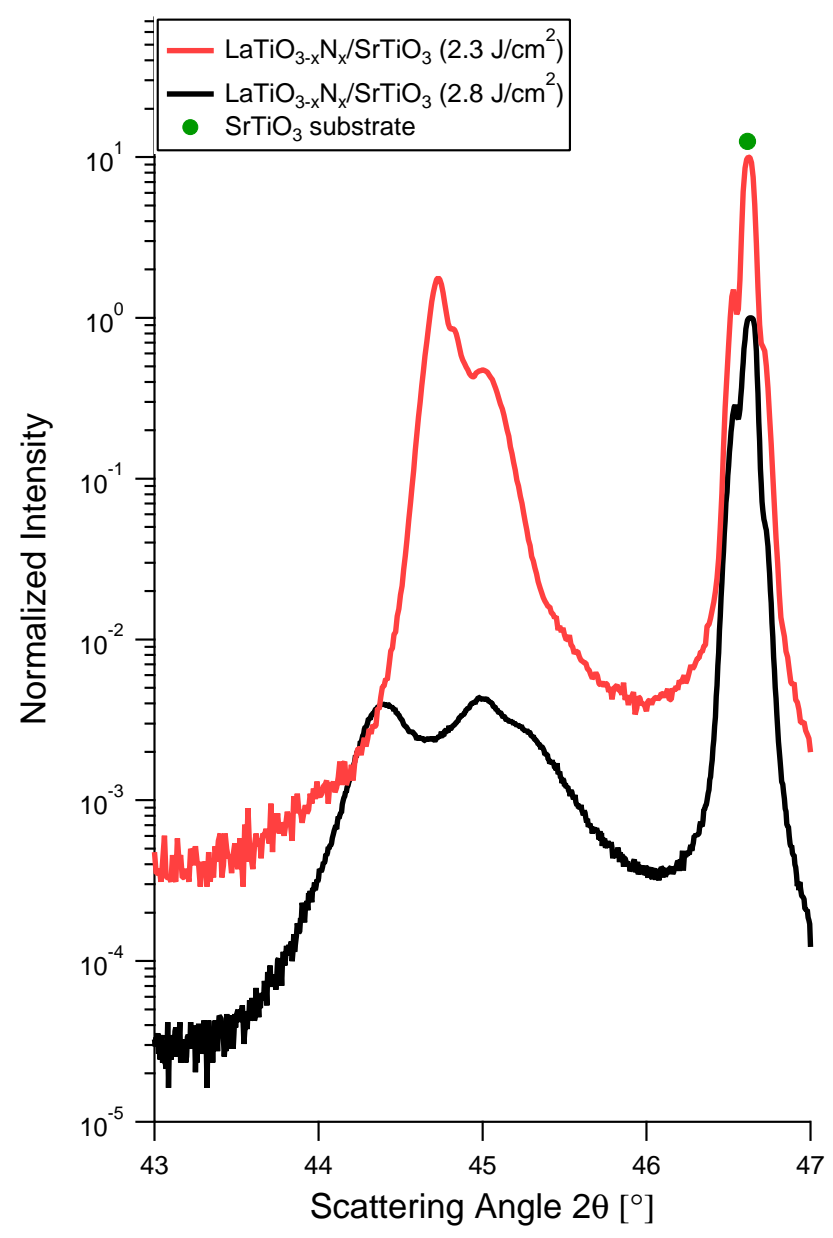

Figure 1: XRD patterns of two representative $\mathrm{LaTiO}_{3-\mathrm{x}} \mathrm{N}_{\mathrm{x}}$ samples grown on $\mathrm{SrTiO}_{3}$ substrates by PRCLA with different laser fluences. 




Figure 2: a) XRD pattern of a polycrystalline $\mathrm{La}_{1.01} \mathrm{Ti}_{0.99} \mathrm{O}_{2.2} \mathrm{~N}_{0.8}$ thin film deposited on TiN-buffered MgO substrate using PRCLA with a laser fluence of $3.7 \mathrm{~J} \mathrm{~cm}^{-2}$. The peak marked with an $\mathrm{X}$ is the $\mathrm{K}_{\beta}$ diffraction reflex from the $\mathrm{MgO}$ substrate. b) Cross section micrograph (fractured surface). c) The corresponding RBS spectrum (black points) analyzed with the RUMP program (red line) and using the $N / O$ ratio determined by ERDA. 


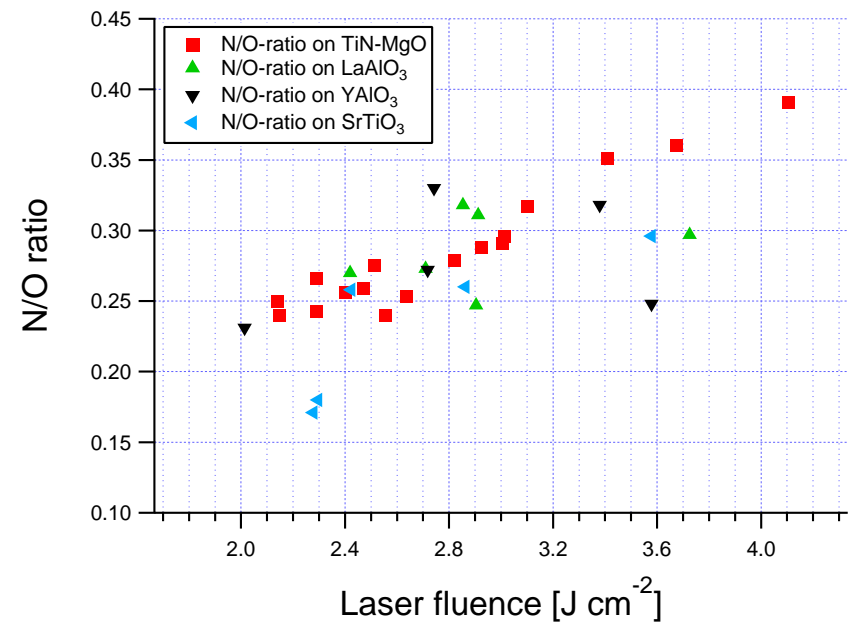

Figure 3: $N / O$ ratio of $\mathrm{LaTiO}_{3-\mathrm{x}} \mathrm{N}_{\mathrm{x}}$ thin films grown on different substrates as a function of laser fluence. Used substrates are $\mathrm{SrTiO}_{3}$ (blue 4 ), $\mathrm{YAlO}_{3}$ (black $\mathbf{v}$ ), $\mathrm{LaAlO}_{3}$ (light green $\boldsymbol{\Delta}$ ), TiN-coated $\mathrm{MgO}$ (red $\boldsymbol{\square}$ ). All thin films are grown at $870{ }^{\circ} \mathrm{C}$ and are $\sim 200 \mathrm{~nm}$ thick. 


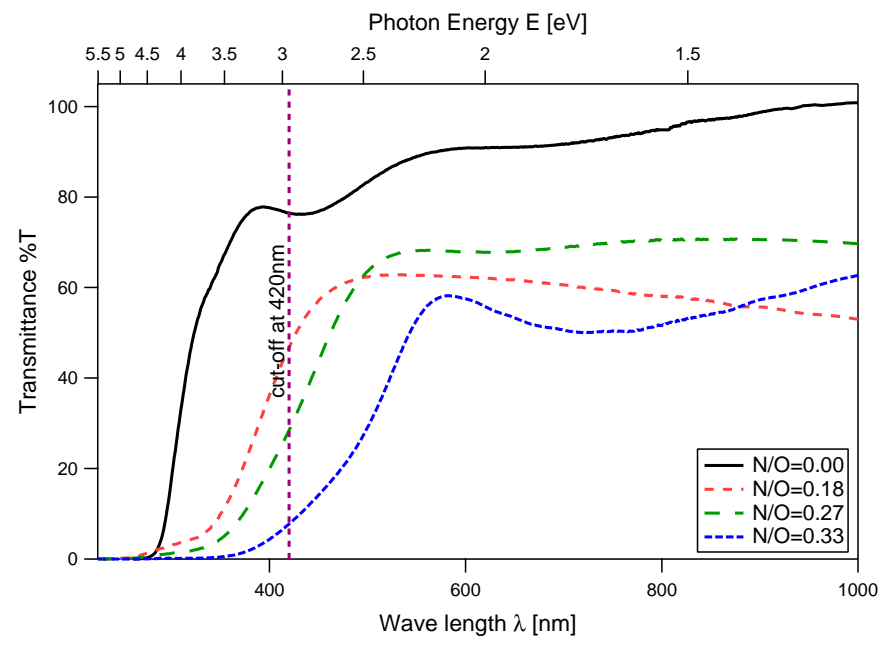

Figure 4: UV-Vis spectra of three $\mathrm{LaTiO}_{3-\mathrm{x}} \mathrm{N}_{\mathrm{x}}$ samples grown on $\mathrm{LaAlO}_{3}$ substrates with different nitrogen content showing the effect on the optical properties of nitrogen substitution into the native oxide. All samples are about $200 \mathrm{~nm}$ thick and therefore interference fringes are observed during the UV-Vis measurement 61. For comparison, the transmittance spectrum of the $\mathrm{La}_{2} \mathrm{Ti}_{2} \mathrm{O}_{7}$ thin film of the same thickness is also shown. 


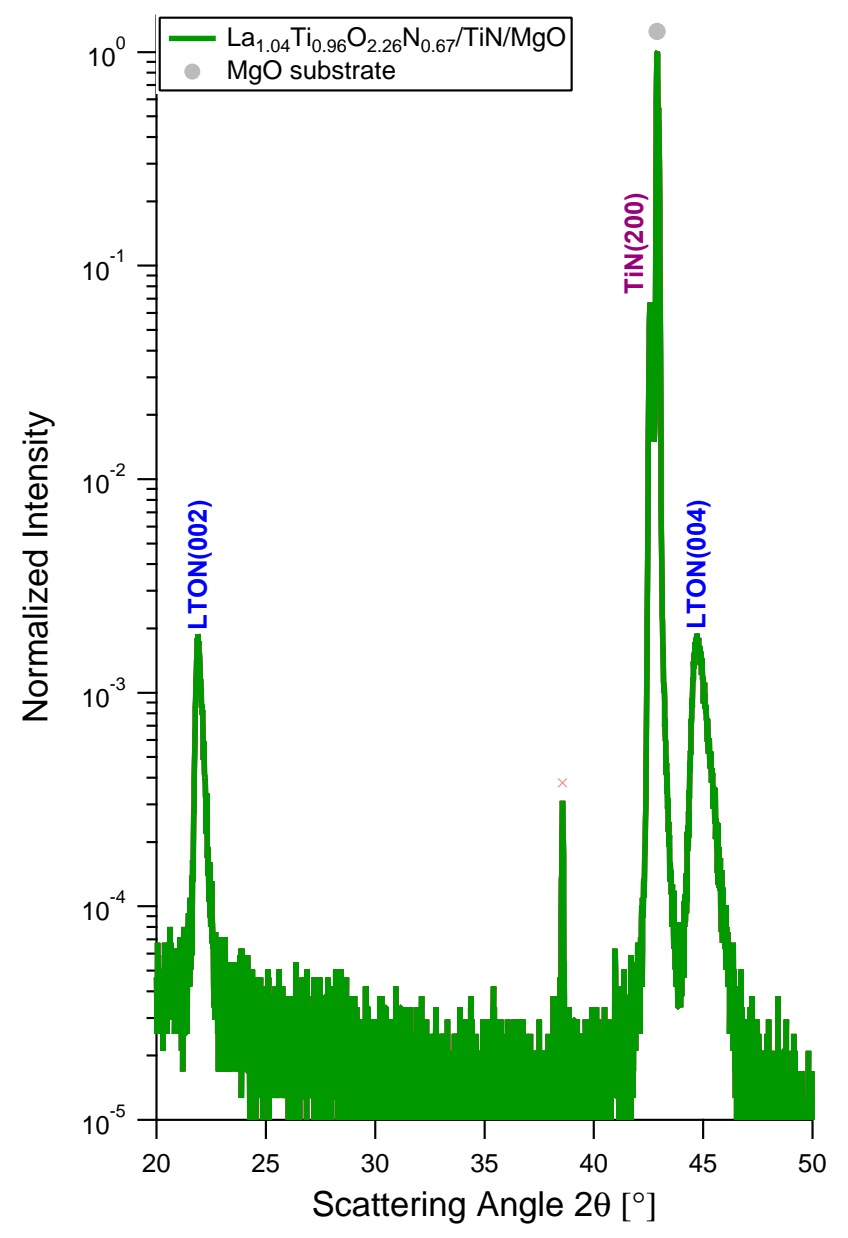

Figure 5: XRD pattern of the epitaxial (001)-oriented $\mathrm{La}_{1.04} \mathrm{Ti}_{0.96} \mathrm{O}_{2.26} \mathrm{~N}_{0.67}$ thin film grown by PRCLA with a laser fluence of $3.0 \mathrm{~J} \mathrm{~cm}^{-2}$. The peak marked with an $\mathrm{X}$ is the $\mathrm{K}_{\beta}$ diffraction reflex from the $\mathrm{MgO}$ substrate. 


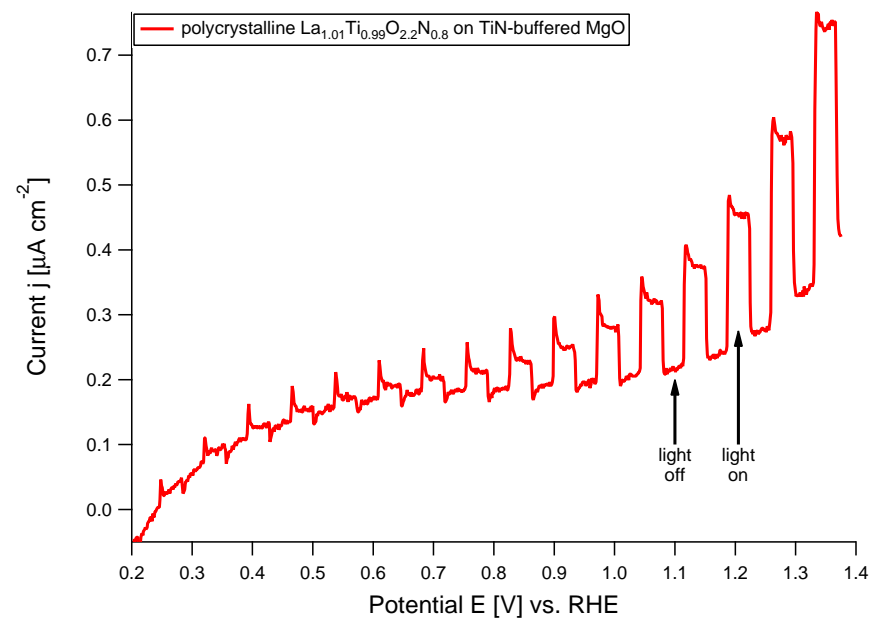

Figure 6: PEC measurement of the polycrystalline $\mathrm{La}_{1.01} \mathrm{Ti}_{0.99} \mathrm{O}_{2.2} \mathrm{~N}_{0.8}$ sample prepared on a TiN-buffered MgO substrate. The measurement was performed under chopped illumination $\left(100 \mathrm{~mW} \mathrm{~cm}^{-2}\right.$ with UV cutoff filter at $\left.\lambda=420 \mathrm{~nm}\right)$ to simultaneously measure the dark current and the photocurrent. The cell was filled with sodium borate electrolyte buffered at $\mathrm{pH}$ of 9 and a linear scan voltammetry was performed at a scan rate of $10 \mathrm{mV} \mathrm{s}^{-1}$. 


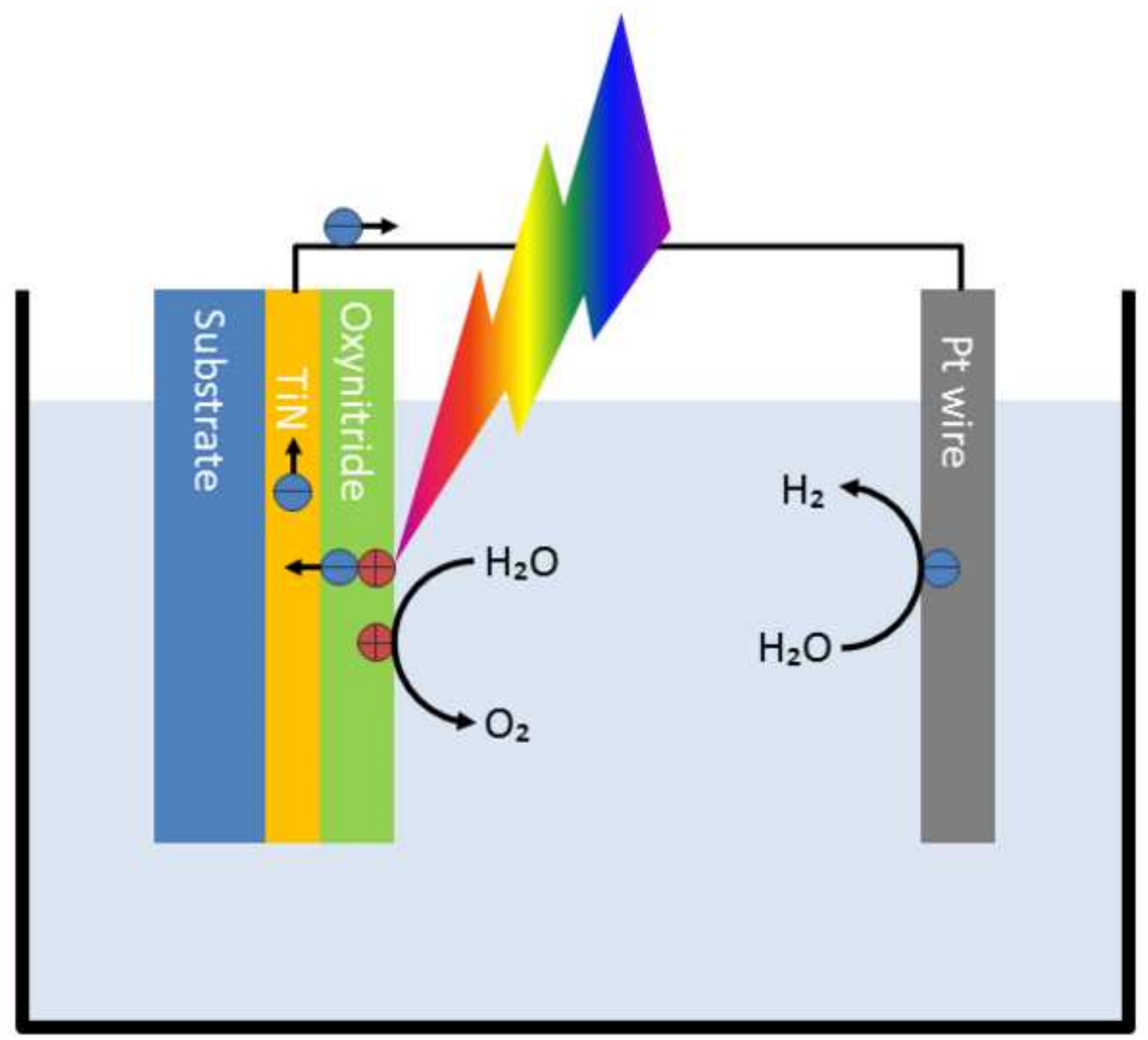

